\title{
Clinical Study Effects of FMO3 Polymorphisms on Pharmacokinetics of
Sulindac in Chinese Healthy Male Volunteers
}

\author{
Yong-Jun Tang, ${ }^{1,2,3}$ Kai Hu, ${ }^{4}$ Wei-Hua Huang, ${ }^{1,2,5}$ Chong-Zhi Wang, ${ }^{5}$ Zhi Liu, ${ }^{5}$ Yao Chen, ${ }^{1,2}$ \\ Dong-Sheng Ouyang, ${ }^{1,2}$ Zhi-Rong Tan, ${ }^{1,2}$ Hong-Hao Zhou, ${ }^{1,2}$ and Chun-Su Yuan ${ }^{5}$ \\ ${ }^{1}$ Department of Clinical Pharmacology, Xiangya Hospital, Central South University, Changsha 410008, China \\ ${ }^{2}$ Institute of Clinical Pharmacology, Hunan Key Laboratory of Pharmacogenetics, Central South University, Changsha 410078, China \\ ${ }^{3}$ Department of Pediatrics, Xiangya Hospital, Central South University, Changsha 410008, China \\ ${ }^{4}$ Department of Neurology, Xiangya Hospital, Central South University, Changsha 410078, China \\ ${ }^{5}$ Tang Center for Herbal Medicine Research, The Pritzker School of Medicine, University of Chicago, 5841 South Maryland Avenue, \\ MC 4028, Chicago, IL 60637, USA
}

Correspondence should be addressed to Wei-Hua Huang; endeavour34852@126.com and Zhi-Rong Tan; tanzr@163.com

Received 31 August 2016; Revised 1 November 2016; Accepted 17 November 2016; Published 26 February 2017

Academic Editor: Heiko Reutter

Copyright (C) 2017 Yong-Jun Tang et al. This is an open access article distributed under the Creative Commons Attribution License, which permits unrestricted use, distribution, and reproduction in any medium, provided the original work is properly cited.

\begin{abstract}
Sulindac is a nonsteroidal anti-inflammatory drug, which is clinically used for the ailments of various inflammations. This study investigated the allele frequencies of FMO3 E158K and E308G and evaluated the influences of these two genetic polymorphisms on the pharmacokinetics of sulindac and its metabolites in Chinese healthy male volunteers. Eight FMO3 wild-type (FMO3 HHDD) subjects and seven FMO3 homozygotes E158K and E308G mutant (FMO3 hhdd) subjects were recruited from 247 healthy male volunteers genotyped by PCR-RFLP method. The plasma concentrations of sulindac, sulindac sulfide, and sulindac sulfone were determined by UPLC, while the pharmacokinetic parameters of the two different FMO3 genotypes were compared with each other. The frequencies of FMO3 E158K and E308G were 20.3\% and 20.1\%, respectively, which were in line with Hardy-Weinberg equilibrium $\left(D^{\prime}=0.977, r^{2}=0.944\right)$. The mean values of $C_{\max }, \mathrm{AUC}_{0-24}$, and $\mathrm{AUC}_{0-\infty}$ of sulindac were significantly higher in FMO3 hhdd group than those of FMO3 HHDD group $(P<0.05)$, while the pharmacokinetic parameters except $T_{\max }$ of sulindac sulfide and sulindac sulfone showed no statistical difference between the two groups. The two FMO3 mutants were in close linkage disequilibrium and might play an important role in the pharmacokinetics of sulindac in Chinese healthy male volunteers.
\end{abstract}

\section{Introduction}

Sulindac, approved by FDA in 1978, is a nonsteroidal antiinflammatory drug (NSAID), which has clinically been used for the ailments of various inflammations over thirty years [1]. NSAIDs have antipyretic, analgesic, and anti-inflammatory effects by blocking the synthesis of prostaglandins due to the significant inhibition of cyclooxygenase [2]. Sulindac as a prodrug that contains a racemic sulfoxide moiety could be transformed into the active drug, sulindac sulfide, by gut microbiota before absorption, while it could be metabolized by CYP450 in liver after absorption [3]. Subsequently, sulindac sulfide is oxidized to be the inactive metabolite, sulindac sulfone (Figure 1), which is catalyzed by the principal isozyme involved in the main metabolic pathway, flavin-containing monooxygenase subtype 3 (FMO3) [3, 4]. The normal product of FMO 3 contains 532 amino acids (Molecular weight, $c a$. $60 \mathrm{kDa})[5,6]$, which is responsible for the oxidation of many clinically important drugs such as cimetidine [7], ranitidine [8], benzydamine [9], clozapine [10], and sulindac [11].

To date, approximate forty single nucleotide polymorphisms (SNPs) have been identified in the FMO3 gene, ten of which cause altered enzyme activity and may affect its substrates metabolism [12]. FMO3/Lys ${ }^{158}$ and FMO3/Gly ${ }^{308}$ have been identified as two predominant FMO3 genetic mutants, which could inactivate the enzyme and the polymorphisms of which appeared at approximately $18 \%$ of both mutations in Korean population [13]. In vivo assay demonstrated that the FMO3 activity showed no difference between heterozygous and homozygous volunteers carrying either one of the two 


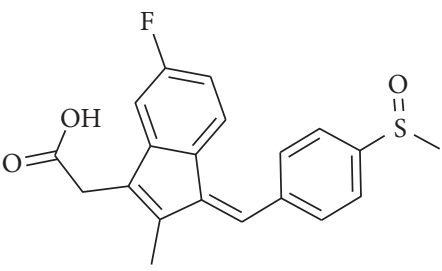

(a)

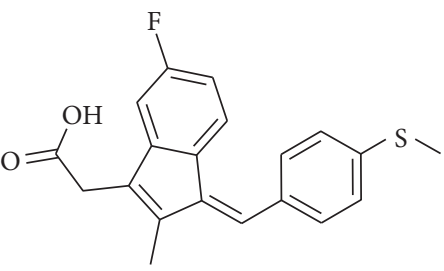

(b)

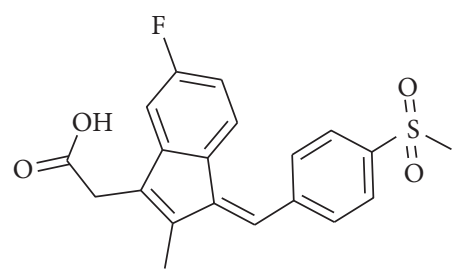

(c)

FIgUre 1: Chemical structure of sulindac (a) and its metabolites and sulindac sulfide (b) and sulindac sulfone (c).

alleles, while its activity would be significantly diminished in the subjects who were either heterozygous or homozygous with both alleles [13]. Additionally, it was reported that a mild symptom of trimethylaminuria (TMAU) could be triggered by a TMA challenge test in patients who were homozygous with FMO3 Lys $^{158} / \mathrm{Gly}^{308}$ [14]. Therefore, the activity of FMO3 may be weakened among individuals who are homozygous or heterozygous with both mutations. Soxygenation of sulindac sulfide was more significantly inhibited by methimazole in human liver microsomes genotyped as homozygous FMO3 [Glu158Lys; Glu308Gly] gene compared to that genotyped as wild-type or heterozygote. As well, the tendency was observed in the recombinant including wild-type and FMO3 [Glu158Lys; Glu308Gly] genes [15, 16]. To date, it is unknown whether FMO3 polymorphisms have effects on pharmacokinetics of sulindac in Chinese population.

In this study, we aimed to investigate the allele frequencies of FMO3 E158K and E308G in Chinese healthy population and evaluate the effects of the two FMO3 genetic polymorphisms on pharmacokinetics of sulindac as well as its metabolites in Chinese healthy male volunteers.

\section{Experimental}

2.1. Subjects. The subjects were restricted to be between 18 and 23 years old with standard body mass index between 20 and $25 \mathrm{~kg} / \mathrm{m}^{2}$. Fifteen healthy male subjects (age: $20.5 \pm 2.1$ years; height: $175.5 \pm 8.6 \mathrm{~cm}$; weight: $65.5 \pm 5.5 \mathrm{~kg}$.) were recruited in this study according to the clinical protocol. All the healthy subjects were requested to sign the informed consent after the assessments of physical examination, medical history, electrocardiogram, and standard laboratory tests including blood cell, urinalysis, and biochemical profile. All of the volunteers were required to abstain from alcohol and medication and could only take standard diets during the study. The subjects who had taken antibiotics in three weeks before this study could not be recruited. The study protocol was approved by the Ethics Committee of Xiangya School of Medicine, Central South University, Hunan province, China.

2.2. Study Design. This project was an open-label, randomized, and single-period study. Human plasma was collected from healthy male subjects who were recruited in this pharmacokinetic study. The test drug named Zulida (sulindac tablets; 100 mg per tablet; batch number 060301) was supplied by Ningbo Team Pharm Co., Ltd. (Hangzhou, China). After fasting overnight, each subject was administered with a single oral dose of sulindac tablets $(200 \mathrm{mg})$ with $100 \mathrm{~mL}$ water. Meanwhile, $5 \mathrm{~mL}$ of venous blood samples was collected into an EDTA (K2)-containing tubes from a forearm vein immediately before dosing $(0 \mathrm{~h})$ and at $10 \mathrm{~min}, 20 \mathrm{~min}$, $30 \mathrm{~min}, 45 \mathrm{~min}, 1 \mathrm{~h}, 1.5 \mathrm{~h}, 2 \mathrm{~h}, 4 \mathrm{~h}, 6 \mathrm{~h}, 8 \mathrm{~h}, 10 \mathrm{~h}, 12 \mathrm{~h}, 24 \mathrm{~h}$, and $36 \mathrm{~h}$ after drug administration. The blood samples were centrifuged at $3000 \mathrm{RPM}$ for $10 \mathrm{~min}$. The collected plasma samples were stored at $-40^{\circ} \mathrm{C}$ until analysis. The subjects were allowed to have meal in $4 \mathrm{~h}$ after oral administration with sulindac tablets.

2.3. Genotyping. In order to screen FMO3 E158K and E308G polymorphisms in this study, 247 healthy Chinese male volunteers were tested to find the subjects in the defined genotypes. Consequently, fifteen healthy male Chinese subjects were totally enlisted, including eight homozygous subjects with the wild-type allele (FMO3 HHDD) and seven homozygous volunteers with the mutant allele (FMO3 hhdd).

The FMO3/Lys ${ }^{158}$ allele was assigned by the lowercase " $h$ " which was identified by the HinFI restriction enzyme while the FMO3/Glu ${ }^{158}$ was assigned by the corresponding uppercase "H"; the FMO3/Gly ${ }^{308}$ allele was assigned by the lowercase "d" which was identified by DraII, and the FMO3/Glu ${ }^{308}$ was designated by an uppercase "D"; genomic DNA was extracted from $5 \mathrm{~mL}$ peripheral blood by standard phenolchloroform method. The wild-type allele (FMO3 HHDD) and the homozygous mutant allele (FMO3 hhdd) were identified according to polymerase chain reaction-restriction fragment length polymorphism (PCR-RFLP) as described previously with slight modification $[17,18]$. The PCR-RFLP experimental conditions were provided in Table 1, and the Macrorestriction Maps of FMO3 E157K and E308G were shown in Figures 2 and 3 , respectively.

2.4. Analytical Assay. Plasma concentrations of sulindac and its metabolites, sulindac sulfide and sulindac sulfone, were quantified by ultraperformance liquid chromatography tandem Photodiode Array (UPLC-PDA) [19]. The analytes were directly extracted by dichloromethane from the collected human plasma using a liquid-liquid extraction method. The chromatographic separation was achieved on Waters Acquity UPLC installed with a Waters Acquity UPLC BEH C18 column $(2.1 \times 50 \mathrm{~mm}$ i.d., $1.7 \mu \mathrm{m})$ within 5 minutes. The mobile phase consisting of ammonium formate buffer 
TABLE 1: PCR-RFLP experiment design of E158K and E308G.

\begin{tabular}{|c|c|c|c|}
\hline Genetic variance & Primers & Restriction endonuclease & Alleles \\
\hline $15167 \mathrm{G}>\mathrm{A}(\mathrm{E} 158 \mathrm{~K})$ & $\begin{array}{c}5^{\prime} \text {-CTGTCTTTGATGCTGTAATGG-3' } \\
5^{\prime} \text {-CAGAAGCGACTGTGAATAG-3' }\end{array}$ & HinfI & $\mathrm{H}, \mathrm{h}$ \\
\hline $21443 \mathrm{~A}>\mathrm{G}(\mathrm{E} 308 \mathrm{G})$ & $\begin{array}{l}5^{\prime} \text {-AACAGGGAACTGGGCATAAG-3' } \\
\text { 5'-ATTGTCACTGGCATTCATCTTC-3 }^{\prime}\end{array}$ & DraII (EcoO109I) & $\mathrm{D}, \mathrm{d}$ \\
\hline
\end{tabular}

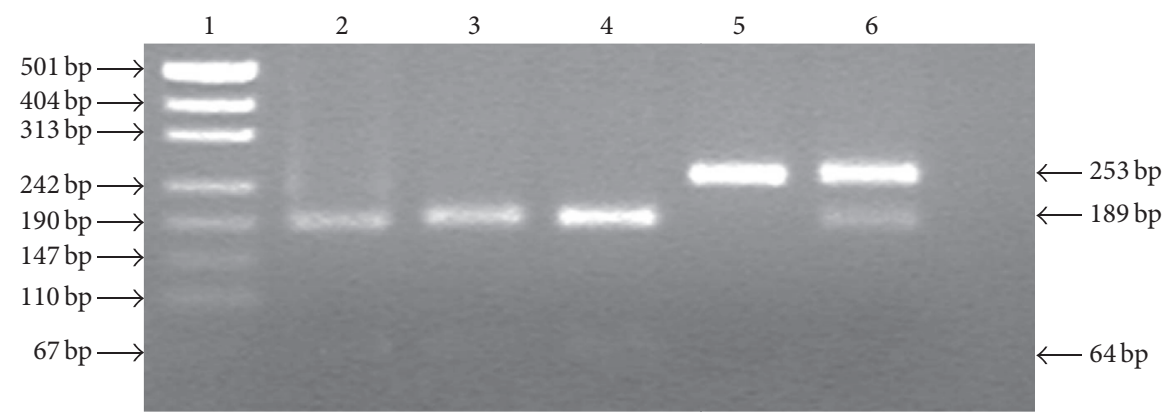

FIGURE 2: Agarose gel electrophoresis of FMO3 E158K after PCR-RFLP. DNA 500 bp marker ladder (Lane 1); wild homozygous, PCR products digested to $189 \mathrm{bp}$ and $64 \mathrm{bp}$ band (Lanes 2, 3, and 4); mutant homozygote (Lane 5); heterozygote (Lane 6).

(20 $\mathrm{mM})$ containing $1 \%$ acetic acid and acetonitrile was used for gradient elution. The flow rate was $0.4 \mathrm{~mL} / \mathrm{min}$ and the monitor wavelength for PDA detection was set at $328 \mathrm{~nm}$.

2.5. Statistical Analysis. The areas under the plasma concentration-time curve (AUC) of sulindac, sulindac sulfide, and sulindac sulfone were calculated according to the linear trapezoidal rule. The elimination rate constant $\left(K_{e}\right)$ was calculated by linear regression of the terminal points using the semilog plot of plasma concentration versus time. The halflife of elimination $\left(T_{1 / 2}\right)$ was calculated by using the formula $T_{1 / 2}=0.693 / K_{e}$. The area under the plasma concentrationtime curve $\left(\mathrm{AUC}_{0-36}\right)$ was calculated by using the linear trapezoidal rule. The area under the plasma concentrationtime curve to time infinity $\left(\mathrm{AUC}_{0-\infty}\right)$ was yielded from the calculations: $\mathrm{AUC}_{0-\infty}=\mathrm{AUC}_{0-36}+C_{36} / K_{e}$, in which $C_{36}$ was the pose-dose plasma concentration of the drug at $36 \mathrm{~h}$.

The pharmacokinetic parameters were calculated by Drug and Statistics for Windows (DAS ver1.0) software. Data were presented as mean \pm SE or SD of at least three independent experiments performed for each sample analysis. The pharmacokinetic parameters between the two genotyped groups were evaluated by Student's $t$-test. Data analysis was performed by the SPSS 19.0 software for windows (Chicago, IL, USA). Values of $P<0.05$ were considered to be statistically significant.

\section{Results}

Before the pharmacokinetics study, previous data showed that the frequency of the FMO3 hhdd genotype was 0.046 in Korean populations, much higher than that in White populations [20]. In this study, we found that FMO3 E158K and E308G were in close linkage disequilibrium in Chinese and the LD between these two SNPs were as strong as that in Koreans. Meanwhile, it was reported that FMO3 hhdd genotype has a frequency of 0.047 in Chinese populations similar to those previously calculated in Korean populations [21]. Under the lifestyle education, our pilot observations on the subjects with the two different genotypes [21] showed that the effective rate was approximately $80 \%$. Thus, we need to enroll 212 subjects to ensure an $80 \%$ power to detect that the drug group has significant effects compared to that of wild-genotype volunteers. Considering factors such as dropouts, 247 subjects were recruited for the screening, while eight homozygous subjects with the wild-type allele (FMO3 HHDD) and seven homozygous volunteers with the mutant allele (FMO3 hhdd) were subjected in this study.

Our previous report on the determination of sulindac and its metabolites, sulindac sulfide and sulindac sulfone, in human plasma had demonstrated that the developed method was precise, accurate, and stable for quantification of these analytes [19]. The extraction efficiencies of sulindac, sulindac sulfide, and sulindac sulfone were all $>75 \%$. The upper limits of quantification were $104.1 \mu \mathrm{g} / \mathrm{mL}$ for sulindac, $117.6 \mu \mathrm{g} / \mathrm{mL}$ for sulindac sulfide, and $104.4 \mu \mathrm{g} / \mathrm{mL}$ for sulindac sulfone in this assay. The lower limits of quantification were $813.4 \mathrm{ng} / \mathrm{mL}, 918.8 \mathrm{ng} / \mathrm{mL}$, and $815.6 \mathrm{ng} / \mathrm{mL}$ for sulindac, sulindac sulfide, and sulindac sulfone, respectively. The analytical recoveries for all the three analytes were in the range of $85 \%-110 \%$, while the interday and intraday precision for the three analytes were all less than $15 \%$.

Significant linkage disequilibrium (LD) was apparent between the two alleles in Chinese population $\left(D^{\prime}=0.9774\right.$, $r^{2}=0.9442$, Table 2). Eight subjects with FMO3 HHDD genotype and seven subjects with FMO3 hhdd genotype were randomly chosen and enrolled in the pharmacokinetic study of sulindac. With a single $200 \mathrm{mg}$ oral dose of sulindac tablets, the pharmacokinetic parameters of sulindac were significantly different between FMO3 HHDD homozygote and FMO3 hhdd homozygote. $\mathrm{AUC}_{0-\infty}$ value of sulindac in 


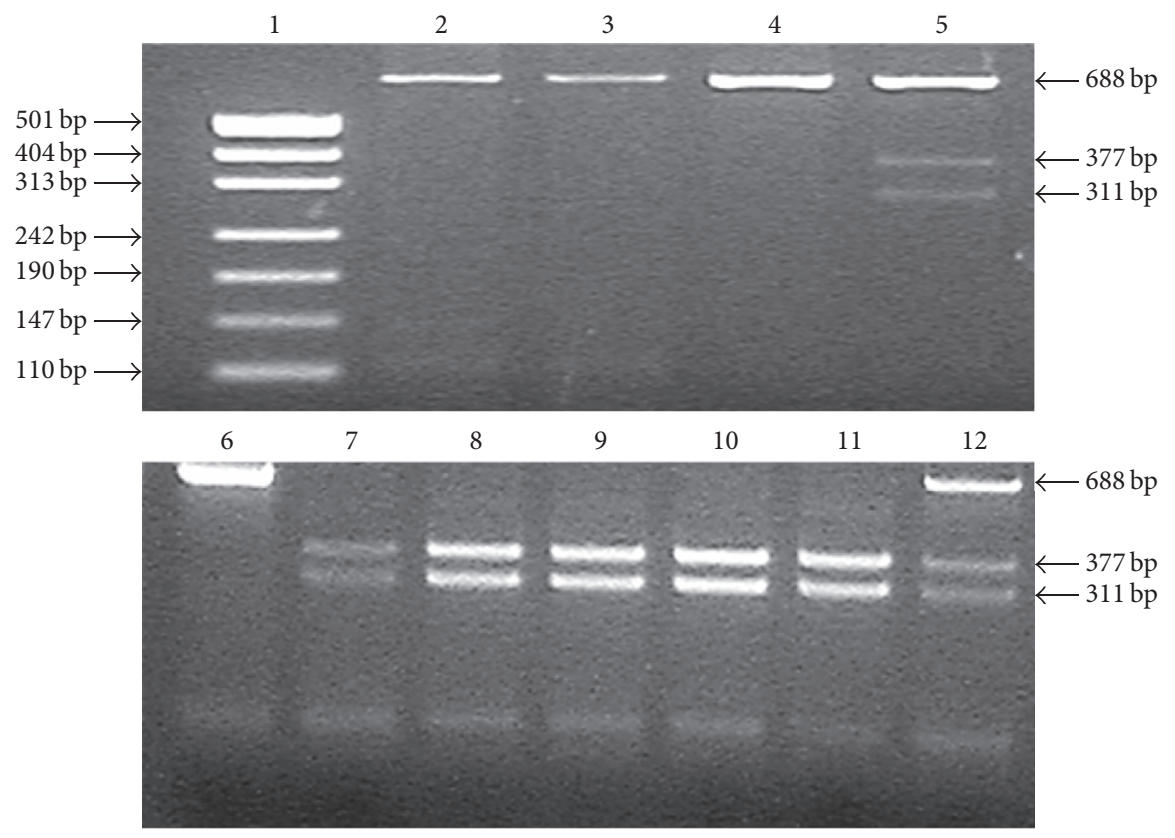

FIGURE 3: Agarose gel electrophoresis of FMO3 E308G after PCR-RFLP. DNA 500 bp marker ladder (Lane 1); heterozygote (Lanes 2, 3 , 4, and 6); mutant homozygote, PCR products digested to $377 \mathrm{bp}+311 \mathrm{bp}$ band (Lanes 7, 8, 9, 10, 11, and 12).

TABLE 2: Analysis of linkage disequilibrium of E158K and E308G.

\begin{tabular}{lcc}
\hline Genotype & Number & $(\%)$ \\
\hline HHDD & 174 & $(63.5)$ \\
HHDd & 2 & $(0.7)$ \\
HHdd & 0 & $(0)$ \\
HhDD & 3 & $(1.7)$ \\
HhDd & 82 & $(29.9)$ \\
Hhdd & 0 & $(0)$ \\
hhDD & 0 & $(0)$ \\
hhDd & 0 & $(0)$ \\
hhdd & 13 & $(4.7)$ \\
Total & 247 & $(100.0)$ \\
$D^{\prime}$ & 0.977 & \\
$r^{2}$ & 0.944 & \\
\hline$D^{\prime}>0.5$ and $r^{2}>1 / 3$, which showed linkage disequilibrium. (http:// \\
analysis.bio-x.cn/myAnalysis.php).
\end{tabular}

the FMO3 hhdd group was much higher than that in the FMO3 HHDD group $(41.88 \pm 17.37$ versus $27.93 \pm 8.85 \mu \mathrm{g} \cdot \mathrm{h} /$ $\mathrm{mL}, P<0.05) . C_{\max }$ value of sulindac in FMO3 hhdd group was higher than that in FMO3 HHDD group (11.87 \pm 4.80 versus $6.95 \pm 3.28 \mu \mathrm{g} / \mathrm{mL}, P<0.05) . T_{1 / 2}$ value of sulindac FMO3 hhdd group was higher than that in FMO3 HHDD group $(4.46 \pm 1.30$ versus $4.01 \pm 1.24 \mathrm{~h}, P<0.05)$. Meanwhile, $C_{\max }, T_{1 / 2}, \mathrm{AUC}_{0-24 \mathrm{~h}}$, and $\mathrm{AUC}_{0-\infty}$ of sulindac sulfide and sulindac sulfone in FMO3 hhd group were not significantly lower than those in the FMO3 HHDD group. The main pharmacokinetic parameters of sulindac, sulindac sulfide, and sulindac sulfone after oral administration of $200 \mathrm{mg}$ sulindac tablets were also calculated, while the main pharmacokinetic parameters of sulindac, sulindac sulfide,
TABLE 3: Mean pharmacokinetic parameters of sulindac, sulindac sulfide, and sulindac sulfone for FMO3 $\operatorname{HHDD}(n=8)$ and FMO3 hhdd $(n=7)$.

\begin{tabular}{lcc}
\hline & FMO3 HHDD & FMO3 hhdd \\
\hline Sulindac & & \\
$\mathrm{AUC}_{0-\infty}\left(\mathrm{ng} \cdot \mathrm{h} \cdot \mathrm{mL}^{-1}\right)$ & $27.93 \pm 8.85$ & $41.88 \pm 17.37^{*}$ \\
$\mathrm{AUC}_{0-24 \mathrm{~h}}\left(\mathrm{ng} \cdot \mathrm{h} \cdot \mathrm{mL}^{-1}\right)$ & $22.82 \pm 7.87$ & $35.16 \pm 9.60^{*}$ \\
$C_{\max }\left(\mathrm{ng} \cdot \mathrm{mL}^{-1}\right)$ & $6.95 \pm 3.28$ & $11.87 \pm 4.80^{*}$ \\
$T_{\max }(\mathrm{h})$ & $1.82 \pm 1.07$ & $1.64 \pm 0.48$ \\
$T_{1 / 2}(\mathrm{~h})$ & $4.01 \pm 1.24$ & $4.46 \pm 1.30^{*}$ \\
Sulindac sulfide & & \\
$\mathrm{AUC}_{0-\infty}\left(\mathrm{ng} \cdot \mathrm{h} \cdot \mathrm{mL}^{-1}\right)$ & $43.69 \pm 15.23$ & $49.67 \pm 25.92$ \\
$\mathrm{AUC}_{0-24 \mathrm{~h}}\left(\mathrm{ng} \cdot \mathrm{h} \cdot \mathrm{mL}^{-1}\right)$ & $32.93 \pm 15.59$ & $32.22 \pm 13.56$ \\
$C_{\max }\left(\mathrm{ng} \cdot \mathrm{mL}^{-1}\right)$ & $32.93 \pm 15.59$ & $32.22 \pm 13.56$ \\
$T_{\max }(\mathrm{h})$ & $2.43 \pm 1.59$ & $3.07 \pm 1.17^{*}$ \\
$T_{1 / 2}(\mathrm{~h})$ & $10.18 \pm 3.28$ & $9.77 \pm 3.97$ \\
Sulindac sulfone & & \\
$\mathrm{AUC}_{0-\infty}\left(\mathrm{ng} \cdot \mathrm{h} \cdot \mathrm{mL}^{-1}\right)$ & $65.64 \pm 16.98$ & $65.69 \pm 24.34$ \\
$\mathrm{AUC}_{0-24 \mathrm{~h}}\left(\mathrm{ng} \cdot \mathrm{h} \cdot \mathrm{mL}^{-1}\right)$ & $48.18 \pm 0.31$ & $42.76 \pm 14.67$ \\
$C_{\max }\left(\mathrm{ng} \cdot \mathrm{mL}^{-1}\right)$ & $3.43 \pm 1.19$ & $3.69 \pm 1.61$ \\
$T_{\max }(\mathrm{h})$ & $2.57 \pm 0.98$ & $2.86 \pm 1.07$ \\
$T_{1 / 2}(\mathrm{~h})$ & $12.19 \pm 2.44$ & $10.62 \pm 2.31$ \\
\hline
\end{tabular}

$C_{\max }$, maximum plasma concentration; AUC, area under plasma concentration-time curve; $T_{\max }$, time of maximum plasma concentration; $T_{1 / 2}$, halflife of elimination. ${ }^{*} P<0.05$.

and sulindac sulfone in different genotypes were provided in Table 3.

The mean plasma concentration-time curves of sulindac, sulindac sulfide, and sulindac sulfone were plotted. The mean plasma concentration-time curves for sulindac, sulindac 


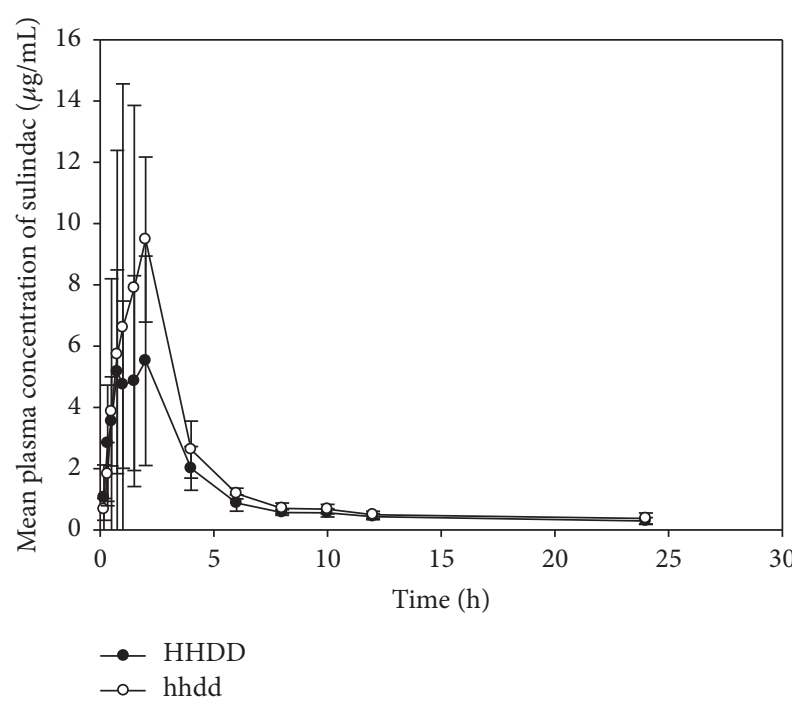

(a)

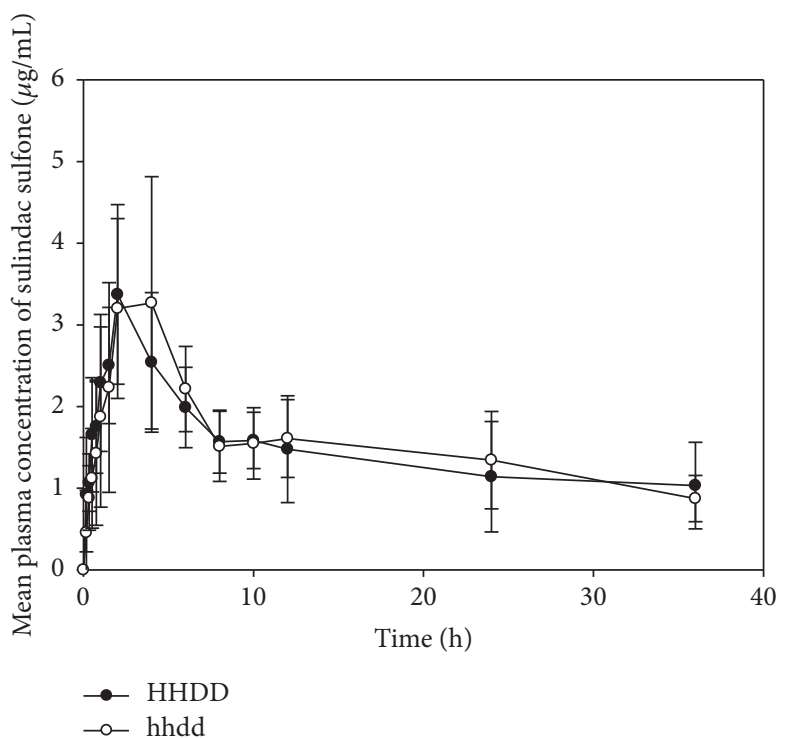

(b)

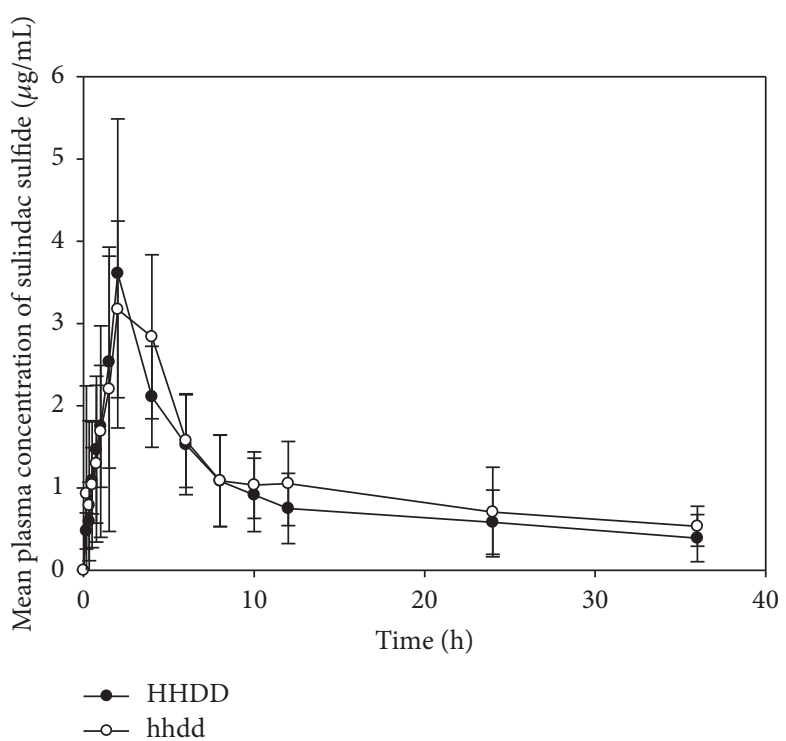

(c)

FIGURE 4: The mean plasma concentration-time curves of sulindac (a), sulindac sulfide (b), and sulindac sulfone (c) in FMO3 HHDD homozygotes $(n=8)$ and FMO3 hhdd homozygotes $(n=7)$ after a single oral dose of $200 \mathrm{mg}$ sulindac tablets.

sulfide, and sulindac sulfone in FMO3 HHDD group and FMO3 hhdd group were described in Figure 4.

\section{Discussion}

In this study, 247 Chinese healthy volunteers were recruited for FMO3 hhdd genotype analysis. Previous data showed that the frequency of the FMO3 hhdd genotype was 0.046 in Korean populations, which was much higher than that in White populations [20]. In Mongolian race, it was the first time to discover that LD between FMO3 E158K and E308G in Chinese was as strong as Koreans. Fortunately, we identified thirteen subjects with the FMO3 hhdd genotype with a frequency of 0.047 that was similar to those previously reported in Korean populations [20].

The effects of genetic polymorphisms of FOM3 on substrates metabolism had already been reported such as the Michaelis constant $\left(K_{m}\right)$ for trimethylamine, which was cautiously increased in the homozygous FMO3 Lys ${ }^{158}$ mutants compared with that in the wild-type carriers [22]. Additionally, individuals with heterozygous or homozygous $\mathrm{Lys}^{158} / \mathrm{Gly}^{308}$ alleles had significantly decreased the plasma concentration of trimethylamine $N$-oxygenation after oral administration of trimethylamine [14]. Moreover, both mutant alleles had been found in patients with trimethylaminuria, who had difficulties in metabolizing trimethylamine [23]. Hereby, it was reasonably concluded that the 
genetic polymorphisms of FMO3 would have effects on the metabolizing of clinical drugs as FMO3's substrates.

NSAIDs including sulindac are effectively chemopreventive agents in some autosomal dominant genetic diseases [24], such as familial adenomatous polyposis (FAP) with an approximate incidence rate of $1 / 10,000$, in which a great number of adenomatous polyps are generated mainly in the epithelium of the large intestine [25]. Sulindac is a prodrug that is converted by gut microbacteria or CYP450 into its active metabolite, sulindac sulfide, which could be catalyzed into its inactive product by FMO3 [11, 26]. To date, no study pertinent to the pharmacokinetics of sulindac, sulindac sulfide, and sulindac sulfone associated with FMO3 genotype in healthy subjects has been reported so that FMO3 should be considered to be the critical factor for the clinical effect of sulindac $[27,28]$. Importantly, the FMO3 SNPs of E158K and E308G mutations were able to significantly increase the efficacy of sulindac.

This study evidenced that FMO3 polymorphism might have a considerable impact on the pharmacokinetics of sulindac, rather than that of sulindac sulfide and sulindac sulfone. FMO3 HHDD and hhd groups exhibited significantly different pharmacokinetic parameters of sulindac. $C_{\text {max }}, T_{\max }, \mathrm{AUC}_{0-24}$, and $\mathrm{AUC}_{0-\infty}$ of sulindac were much higher in the seven homozygous FMO3 hhdd subjects than those in the eight homozygous FMO3 HHDD subjects $(P<$ 0.05), while differences regarding $C_{\max }, T_{1 / 2}, \mathrm{AUC}_{0-24}$, and $\mathrm{AUC}_{0-\infty}$ of sulindac sulfone and sulindac sulfide were not significant in these two groups. $T_{\max }$ of sulindac sulfide was lower in FMO3 hhdd subjects than in FMO3 HHDD subjects $(P<0.05)$. No significant difference was observed in the relative $T_{\max }$ values of sulindac sulfone between the two genotyped groups, because sulindac sulfone was absorbed from gastrointestinal tract to keep away from the influence of FMO3. The exposure of sulindac was definitely higher in FMO3 hhdd homozygous subjects than in FMO3 HHDD homozygous subjects, which was clearly discriminated by the remarkable discrepancy that $\mathrm{AUC}_{0-\infty}$ value in $\mathrm{FMO} 3$ hhdd carriers was much higher. The results indicated that mutants with FMO3 hhdd genotype could decrease FMO3 enzyme activity, which could change the in vivo biotransformation of sulindac. Though the mutants showed the tendency to reduce the metabolism of sulindac sulfide and sulindac sulfone as manifested by the data of AUC and $C_{\max }$, the differences were not statistically significant. The actual effects of different FMO3 genotypes on sulindac sulfide and sulindac sulfone need further study, because the secondary metabolism and transportation on the two metabolites were unknown and the sample size was limited in this study.

The FMO3 homozygous Lys ${ }^{158} / \mathrm{Gly}^{308}$ carriers had diminished the enzyme activity in catabolizing sulindac. Similar changes were also observed in the pharmacokinetics of benzydamine and ranitidine [20]. It was concluded that FMO3 mutant allele coding proteins with impaired activity ultimately resulted in changes of the clinical consequence for FMO3 substrates. Therefore, the higher plasma concentration of sulindac was possible to increase the toxicity and side effects in FMO3 hhdd subjects. The drug-drug interactions with sulindac should be cautiously considered, when sulindac is coadministered with other FMO3 substrates in patients carrying the FMO3 hhdd mutant.

\section{Conclusion}

FMO3 E158K and E308G polymorphisms played an important role in in vivo metabolism of sulindac. The plasma concentration of sulindac was remarkably higher in the individuals carrying FMO3 hhdd allele than that of the individuals with FMO3 HHDD (wild-type) allele. More attention should be notably paid when sulindac is coadministered with other FMO3 substrates in patients carrying FMO3 hhdd mutant.

\section{Competing Interests}

All the authors declare that they have no conflict of interests.

\section{Authors' Contributions}

Wei-Hua Huang, Zhi-Rong Tan, and Hong-Hao Zhou designed this study. Wei-Hua Huang, Yong-Jun Tang, and Zhi-Rong Tan performed the experiments. Wei-Hua Huang, Yong-Jun Tang, Kai Hu, Chong-Zhi Wang, Yao Chen, and Zhi Liu analyzed the data and illustrated the figures. DongSheng Ouyang and Chun-Su Yuan provided the experimental materials and revised the manuscript. Wei-Hua Huang, YongJun Tang, and Zhi-Rong Tan wrote the manuscript. All authors have read and approved the final manuscript.

\section{Acknowledgments}

This work was supported by the National Natural Scientific Foundation of China (no. 81001471 to Z.-R. Tan, no. 31400306 to W.-H. Huang), General Financial Grant from the China Postdoctoral Science Foundation (no. 2015M570692), Hunan Provincial Natural Science Foundation of China (no. 2015JJ3156), the fundamental research funds for the central universities of Central South University (no. 1681-7608040003), and NIH Grants AT004418 and AT005362.

\section{References}

[1] J. Castellsague, N. Riera-Guardia, B. Calingaert et al., "Individual NSAIDs and upper gastrointestinal complications: a systematic review and meta-analysis of observational studies (the SOS Project)," Drug Safety, vol. 35, no. 12, pp. 1127-1146, 2012.

[2] V. Rollason, C. F. Samer, Y. Daali, and J. A. Desmeules, "Prediction by pharmacogenetics of safety and efficacy of non-steroidal anti-inflammatory drugs: a review," Current Drug Metabolism, vol. 15, no. 3, pp. 326-343, 2014.

[3] D. E. Duggan, L. E. Hare, C. A. Ditzler, B. W. Lei, and K. C. Kwan, "The disposition of sulindac," Clinical Pharmacology and Therapeutics, vol. 21, no. 3, pp. 326-335, 1977.

[4] S. Kitamura, K. Nakatani, K. Ohashi et al., "Extremely high drug-reductase activity based on aldehyde oxidase in monkey liver," Biological and Pharmaceutical Bulletin, vol. 24, no. 7, pp. 856-859, 2001. 
[5] M. S. Motika, J. Zhang, X. Zheng, K. Riedler, and J. R. Cashman, "Novel variants of the human flavin-containing monooxygenase 3 (FMO3) gene associated with trimethylaminuria," Molecular Genetics and Metabolism, vol. 97, no. 2, pp. 128-135, 2009.

[6] H. Yamazaki and M. Shimizu, "Survey of variants of human flavin-containing monooxygenase 3 (FMO3) and their drug oxidation activities," Biochemical Pharmacology, vol. 85, no. 11, pp. 1588-1593, 2013.

[7] J. R. Cashman, "Human flavin-containing monooxygenase: substrate specificity and role in drug metabolism," Current Drug Metabolism, vol. 1, no. 2, pp. 181-191, 2000.

[8] W.-G. Chung, C.-S. Park, H.-K. Roh, W.-K. Lee, and Y.-N. Cha, "Oxidation of ranitidine by isozymes of flavin-containing monooxygenase and cytochrome P450," Japanese Journal of Pharmacology, vol. 84, no. 2, pp. 213-220, 2000.

[9] E. Störmer, I. Roots, and J. Brockmöller, "Benzydamine Noxidation as an index reaction reflecting FMO activity in human liver microsomes and impact of FMO3 polymorphisms on enzyme activity," British Journal of Clinical Pharmacology, vol. 50, no. 6, pp. 553-561, 2000.

[10] C. Sachse, S. Ruschen, M. Dettling et al., "Flavin monooxygenase 3 (FMO3) polymorphism in a white population: allele frequencies, mutation linkage, and functional effects on clozapine and caffeine metabolism," Clinical Pharmacology and Therapeutics, vol. 66, no. 4, pp. 431-438, 1999.

[11] I. M. Hisamuddin, M. A. Wehbi, A. Chao et al., "Genetic polymorphisms of human flavin monooxygenase 3 in sulindacmediated primary chemoprevention of familial adenomatous polyposis," Clinical Cancer Research, vol. 10, no. 24, pp. 83578362, 2004.

[12] S. B. Koukouritaki, M. T. Poch, E. T. Cabacungan, D. G. McCarver, and R. N. Hines, "Discovery of novel flavincontaining monooxygenase 3 (FMO3) single nucleotide polymorphisms and functional analysis of upstream haplotype variants," Molecular Pharmacology, vol. 68, no. 2, pp. 383-392, 2005.

[13] J.-H. Kang, W.-G. Chung, K.-H. Lee et al., "Phenotypes of flavin-containing monooxygenase activity determined by ranitidine $N$-oxidation are positively correlated with genotypes of linked FMO3 gene mutations in a Korean population," Pharmacogenetics, vol. 10, no. 1, pp. 67-78, 2000.

[14] J. Zschocke, D. Kohlmueller, E. Quak, T. Meissner, G. F. Hoffmann, and E. Mayatepek, "Mild trimethylaminuria caused by common variants in FIMO3 gene," Lancet, vol. 354, no. 9181, pp. 834-835, 1999.

[15] S. Nagashima, M. Shimizu, H. Yano et al., "Inter-individual variation in flavin-containing monooxygenase 3 in livers from Japanese: correlation with hepatic transcription factors," Drug Metabolism and Pharmacokinetics, vol. 24, no. 3, pp. 218-225, 2009.

[16] M. Shimizu, H. Yano, S. Nagashima et al., "Effect of genetic variants of the human flavin-containing monooxygenase 3 on N- and S-oxygenation activities," Drug Metabolism and Disposition, vol. 35, no. 3, pp. 328-330, 2007.

[17] D.-C. Hao, J. Sun, B. Furnes et al., "Haplotype frequency distribution and linkage disequilibrium analysis of single nucleotide polymorphisms at the human FMO3 gene locus," Biochemical Genetics, vol. 44, no. 7-8, pp. 391-407, 2006.
[18] D. Hao, J. Sun, B. Furnes et al., "Allele and genotype frequencies of polymorphic FMO3 gene in two genetically distinct populations," Cell Biochemistry and Function, vol. 25, no. 4, pp. 443453, 2007.

[19] W.-H. Huang, L. Shao, S.-X. Li et al., "Simultaneous determination of sulindac and its metabolites sulindac sulfide and sulindac sulfone in human plasma by a sensitive UPLC-PDA method for a pharmacokinetic study," Analytical Methods, vol. 6, no. 13, pp. 4679-4685, 2014.

[20] C.-S. Park, J.-H. Kang, W.-G. Chung et al., "Ethnic differences in allelic frequency of two flavin-containing monooxygenase 3 (FMO3) polymorphisms: linkage and effects on in vivo and in vitro FMO activities," Pharmacogenetics, vol. 12, no. 1, pp. 77-80, 2002.

[21] L.-P. Zhou, Z.-R. Tan, H. Chen et al., "Effect of two-linked mutations of the FMO3 gene on itopride metabolism in Chinese healthy volunteers," European Journal of Clinical Pharmacology, vol. 70, no. 11, pp. 1333-1338, 2014.

[22] J. R. Cashman, B. R. Akerman, S. M. Forrest, and E. P. Treacy, "Population-specific polymorphisms of the human FMO3 gene: significance for detoxication," Drug Metabolism and Disposition, vol. 28, no. 2, pp. 169-173, 2000.

[23] J. R. Cashman and J. Zhang, "Interindividual differences of human flavin-containing monooxygenase 3: genetic polymorphisms and functional variation," Drug Metabolism and Disposition, vol. 30, no. 10, pp. 1043-1052, 2002.

[24] M. Cruz-Correa and F. M. Giardiello, "Familial adenomatous polyposis," Gastrointestinal Endoscopy, vol. 58, no. 6, pp. 885894, 2003.

[25] J.-Q. Sheng, W.-J. Cui, L. Fu et al., "APC gene mutations in Chinese familial adenomatous polyposis patients," World Journal of Gastroenterology, vol. 16, no. 12, pp. 1522-1526, 2010.

[26] I. M. Hisamuddin, M. A. Wehbi, B. Schmotzer et al., "Genetic polymorphisms of Flavin monooxygenase 3 in sulindacinduced regression of colorectal adenomas in familial adenomatous polyposis," Cancer Epidemiology Biomarkers and Prevention, vol. 14, no. 10, pp. 2366-2369, 2005.

[27] D. E. Duggan, K. F. Hooke, E. A. Risley, T. Y. Shen, and C. G. Arman, "Identification of the biologically active form of sulindac," Journal of Pharmacology and Experimental Therapeutics, vol. 201, no. 1, pp. 8-13, 1977.

[28] F. Etienne, L. Resnick, D. Sagher, N. Brot, and H. Weissbach, "Reduction of Sulindac to its active metabolite, sulindac sulfide: assay and role of the methionine sulfoxide reductase system," Biochemical and Biophysical Research Communications, vol. 312, no. 4, pp. 1005-1010, 2003. 

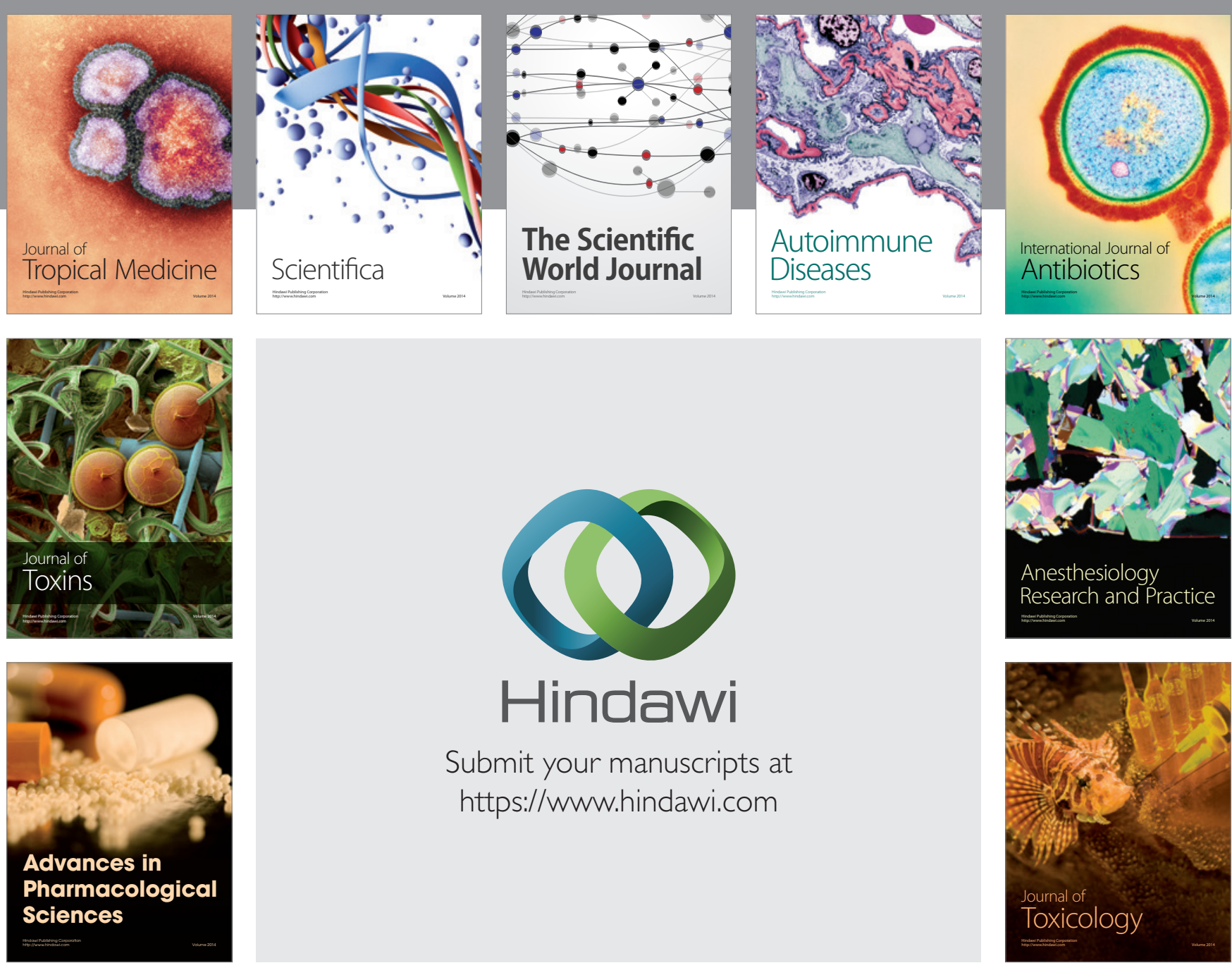

\section{Hindawi}

Submit your manuscripts at

https://www.hindawi.com
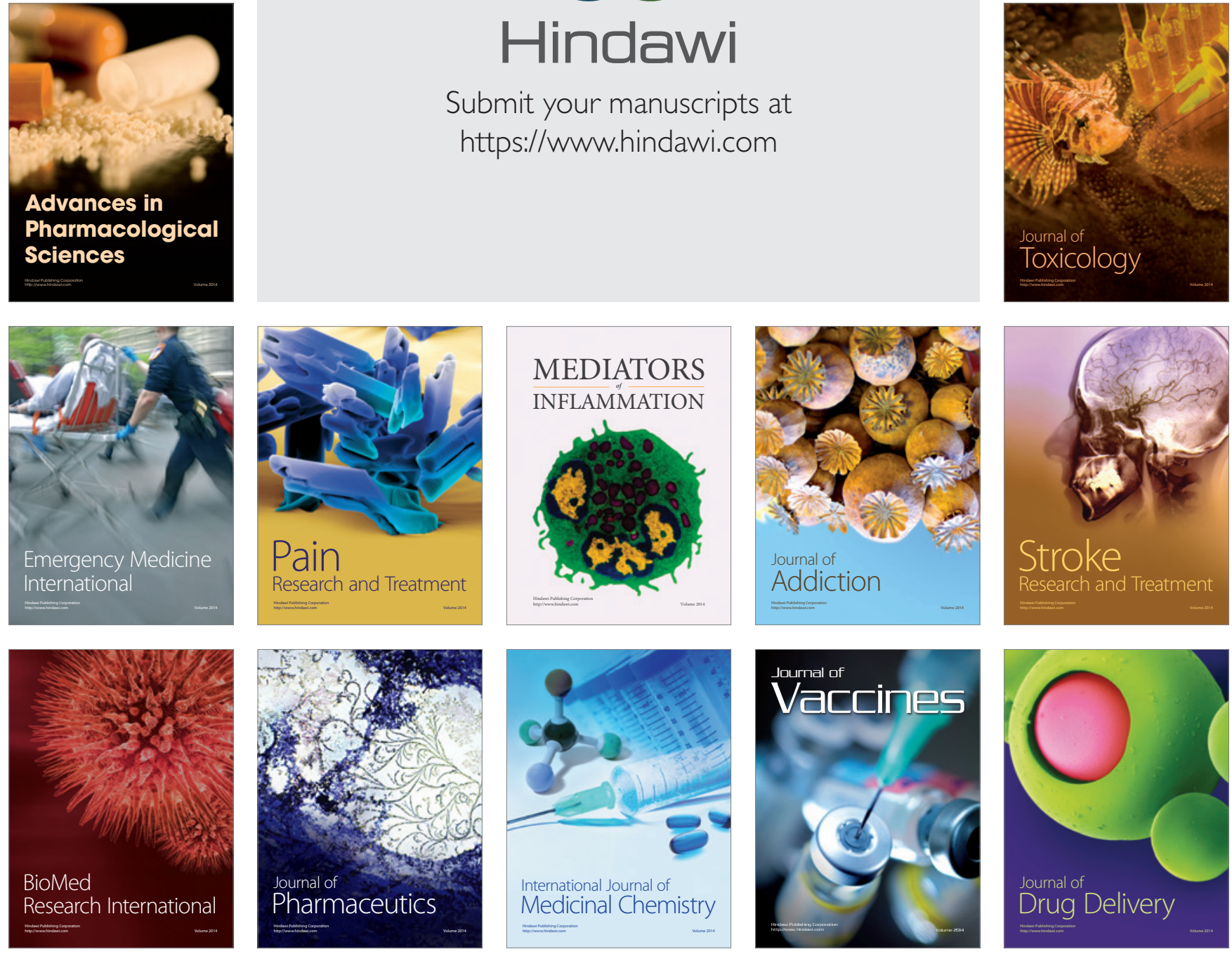